\title{
ÍNDICE DE CAPITAL SOCIAL PARA \\ el Pueblo Mágico de Tapijulapa, Tabasco
}

\section{Rate of Social Capital for the Magic Town of Tapijulapa, Tabasco}

\author{
LUCÍA SANDOVAL NÚNEZZ* \\ María Estela Ortega Rubí**
}

\section{RESUMEN}

El objetivo de esta investigación está dirigida a analizar los eslabones que se enlazan entre la comunidad y los demás actores sociales involucrados en Tapijulapa, Tabasco, como Pueblo Mágico, así como la forma en que estas relaciones influyen en la sustentabilidad de una localidad. Como parte de la metodología se aplicó un cuestionario, el cual fue utilizado para realizar un análisis factorial haciendo uso de la técnica de componentes principales y para hacer un índice del capital social (objeto de este documento), integrado por la relación de los subíndices de confianza y de redes sociales, a fin de proporcionar un análisis detallado del capital social en esta comunidad. Con base en ello, se concluye que en toda sociedad es necesario el establecimiento de sistemas de comunicación eficientes entre los individuos o los grupos con el objetivo de impulsar el trabajo en conjunto, elevar los beneficios esperados del mismo trabajo y, a su vez, el capital social de la comunidad.

Palabras Clave: Relaciones verticales, Capital social, SUSTentabilidad, CONFIANZa Y Redes SOCIALES.

* Universidad Autónoma de Nuevo León, Instituto de Investigaciones Sociales. Correo electrónico: luciasandovalı@hotmail.com.

** Universidad Autónoma de Nuevo León, Instituto de Investigaciones Sociales. Correo electrónico: estela_ort_rubi@hotmail.com. 


\section{Abstract}

The objective of this research is to analyze the links that are woven between the community and the other social actors involved in Tapijulapa, Tabasco, how magical town, as well as the way in which these relationships are able to influence the sustainability of a locality. As part of the methodology was applied a questionnaire, which was used to perform a factor analysis using principal components and to perform an index of social capital (the subject of this document), integrated by the relationship of the subscripts of trust and social networks to provide a detailed analysis of social capital in this community. From this it can be concluded that in every society is necessary to the establishment of efficient communication systems between individuals or groups with the aim of encouraging the work as a whole, raise the expected benefits of the same and, in turn, the social capital of the community.

KEYWORDS: VERTICAL RELATIONSHIPS, SOCIAL CAPITAL, SUSTAINABILITY, TRUST AND SOCIAL NETWORKS.

Recepción: 8 de abril de 2016.

Dictamen 1: 14 de noviembre de 2016.

Dictamen 2: 15 de noviembre de 2016. 


\section{INTRODUCCIÓN}

El turismo es una de las actividades más importantes de la economía en el mundo; sin embargo, ha sido muy susceptible a cambios propiciados por los gustos del turista que siempre se encuentra en demanda de nuevos productos con los que pueda "convivir con las comunidades receptoras, interrelacionarse con la naturaleza, realizar actividades que le impliquen reto físico, búsqueda de emociones significativas, mantenerse en forma, cuidar su salud, realizar actividades al aire libre, entre otras" (SECTUR, 2004, p. 19). Estas expectativas han significado un reto para cada una de las comunidades receptoras de turismo y para las políticas públicas que se han considerado como oportunidades para transformar a la sociedad a través de la calidad de vida de las personas como resultado del crecimiento turístico y de los dividendos que este proporciona.

El turismo es una actividad fundamental en el desarrollo debido a su contribución al producto interno bruto (PIB), a la creación de fuentes de empleo y a la captación de divisas. En México, el turismo conocido como de playa se ha visto afectado recientemente por numerosos factores, entre los que destaca la emergencia de nuevos y llamativos destinos turísticos competidores. Aunado a lo anterior, "en el análisis de la política turística nacional se advierten deficiencias derivadas de la falta de planeación y coordinación, así como de la fragmentación y dispersión de los programas orientados a potenciar esta actividad" (Gris, 2009, p. 6).

Una acción concreta del gobierno federal orientada al fortalecimiento de este sector y a la satisfacción de las exigencias del mercado mundial es la creación de programas regionales como el denominado Pueblos Mágicos, iniciado en 2001, que tiene entre sus objetivos establecer una variada oferta turística hacia el interior del país que encuentre sus cimientos en los atractivos turísticos histórico-culturales de pequeñas localidades.

Para operar el programa de manera exitosa se contempla, en la guía de incorporación y permanencia de Pueblos Mágicos, una serie de actividades dirigidas a este fin; "entre estas se encuentra la coordinación social, interinstitucional e intergubernamental para lo que se requiere de un ejercicio de transversalidad entre todas las instituciones de gobierno involucradas, además de privilegiar la participación de la comunidad receptora, con la finalidad de crear alianzas entre los tres niveles de gobierno, la sociedad local y los actores económicos, empresas y organizaciones" (SECTUR, s/f). 
Dado que el capital social, de acuerdo con Díaz Albertini (2003, p. 247), es aquel que "fomenta la acción colectiva, el autocontrol y la responsabilidad social al acercar entre sí a los seres humanos por medio de la confianza, la reciprocidad y el respeto a las reglas del juego, elementos esenciales para todo proceso de desarrollo, y especialmente el sostenible", queda de manifiesto la relevancia de un estudio que muestre el contexto de las relaciones sociales existentes en los Pueblos Mágicos de México. Por tal razón, en este artículo se exponen los resultados de un estudio dirigido al análisis de las relaciones verticales que se entretejen entre la comunidad y los demás actores sociales de Tapijulapa y la forma en que estas relaciones producen efectos en el desarrollo sustentable de la localidad por medio de fuentes del capital social como la confianza y las redes como una manera de acceder a las oportunidades.

Es palpable la dificultad de hacer un análisis que nos proporcione información de la situación en la que funcionan u operan estas relaciones verticales en los 83 pueblos que cuentan con el nombramiento de Pueblo Mágico, por lo que el presente estudio se ha enfocado en Tapijulapa, Tabasco, como Pueblo Mágico, de donde se ha obtenido información a través de un cuestionario aplicado a los diferentes actores sociales involucrados en este nombramiento. Con los resultados de esta herramienta se realizó un análisis factorial utilizando la técnica de componentes principales y un índice de capital social (objetivo del presente documento). Mediante este índice fueron detectadas áreas de mejora en la investigación, porque se pretende estudiar el capital social en otros Pueblos Mágicos con la finalidad de determinar las estrategias que permitan a las políticas turísticas prestar atención al aprovechamiento del mismo capital en los diferentes Pueblos Mágicos existentes, así como en aquellas pequeñas localidades con vocación turística.

\section{El PROGRAMA PUEBLOS MÁGICOS}

\section{Y LA IMPORTANCIA DEL CAPITAL SOCIAL}

De acuerdo con el diagnóstico del programa presupuestario Programa de Desarrollo Regional Turístico Sustentable y Pueblos Mágicos (s/f), este tiene su origen en los Fondos Mixtos para la Promoción Turística, que apoyaban la promoción, la publicidad y las relaciones públicas en los destinos turísticos ya posicionados en el mercado nacional. En 1998 fueron sustituidos por los Convenios de Coordinación en Materia de Desarrollo y Promoción Turística, cuya denominación fue cambiada 
en 2002 a Convenios de Coordinación en Materia de Reasignación de Recursos (CCRR) orientando los recursos a proyectos de desarrollo en destinos turísticos. En 2013 fue objeto de otro cambio de nombre al de Apoyos para el Desarrollo de la Oferta Turística, en los que los subsidios recibidos no estaban sujetos a reglas de operación. En 2014 recibió una nueva modificación por la cual los subsidios estarían sujetos a reglas de operación. Además, recibió un nuevo nombre: Programa para el Desarrollo Regional Turístico Sustentable (PRODERETUS). En 2015 se creó un nuevo programa presupuestario destinado a apoyar la infraestructura y el equipamiento relativos al Programa Pueblos Mágicos y Destinos Prioritarios, el cual fue fusionado con el anterior en 2016 y fue denominado Programa de Desarrollo Regional Turístico Sustentable y Pueblos Mágicos.

"El programa Pueblos Mágicos está enfocado hacia localidades con cierto grado de desarrollo turístico y con deseos de impulsar al turismo para elevar los niveles de bienestar, aumentar el empleo, fomentar y hacer rentable la inversión, así como fortalecer y optimizar el aprovechamiento racional de los recursos y atractivos naturales y culturales" (SECTUR, s/f). La idea principal de esta política consiste en volver partícipe del proceso de desarrollo a las comunidades receptoras y a la sociedad en su conjunto para el logro de destinos sustentables.

Como hemos podido observar, la inversión pública en este programa para comunidades con vocación turística ha llevado a estas a obtener recursos enfocados a mejorar la infraestructura y servicios, el equipamiento turístico, la creación o fortalecimiento de las rutas turísticas, la creación de sitios de interés turístico y asistencia técnica y servicios relacionados con los proyectos. No obstante, los resultados económicos, sociales y ambientales son desiguales en cada pueblo, debido principalmente a las características físicas, sociales, culturales, económicas, demográficas y políticas que circundan a la localidad en cuestión.

A pesar de que los resultados difieren unos de otros dependiendo de las características del pueblo estudiado, es necesario impulsar la generación de políticas públicas que desde su origen contemplen el capital social existente, una mejor adaptación a este y mejores vínculos entre actores sociales, dado que en este proceso interactúan diversos actores sociales con culturas y conocimientos diversos que dan la pauta a la interdisciplinaridad cuya base es la comunicación y las relación entre las partes para su mejor aprovechamiento.

Los beneficios de la aplicación de este tipo de enfoque se han observado con claridad en Australia, "donde la intervención de varios niveles de gobierno en la mercadotecnia turística y en la administración a nivel regional, así como el peso de 
los pequeños negocios turísticos han propiciado la creación de sistemas turísticos regionales innovadores y exitosos" (Macbeth et al., 2004, p. 508). Estos sistemas turísticos regionales han incluido a la comunidad en su totalidad, la interacción entre visitantes y pobladores locales, los diferentes sectores que proporcionan servicios a los turistas, las condiciones económicas de la región (en la creación de infraestructura que facilite el tránsito a los destinos turísticos y la disponibilidad de los servicios), entre otros. El capital social, político y cultural es la herramienta que ha representado la forma de entender las características de las comunidades contribuyendo al desarrollo sustentable de estas.

Existen además otras investigaciones orientadas a la importancia del capital social como factor de desarrollo en localidades, como la de Prat i Forga (2014), que analiza el capital social generado por todos los actores involucrados en dos destinos que reciben turismo industrial, Cataluña y Escocia. Este estudio tuvo como objetivo confirmar si una mayor dinámica relacional de estas redes sociales corresponde a un mayor grado de desarrollo turístico. Los resultados muestran la necesidad de la elaborar e implementar políticas públicas orientadas al desarrollo del capital social de las comunidades. Prat encontró una relación estrecha entre el capital social de los actores que conforman las redes sociales, la dinámica relacional de las redes y el grado de desarrollo del turismo en el territorio. Por ello, sugiere que los principales actores, como el caso de las administraciones locales, deben involucrarse y generar una serie de redes más compactas enfocadas en la promoción de la cooperación y la difusión de actividades, además de potenciar su labor como intermediarios entre los agentes privados y los demás agentes públicos.

Mientras que Merinero (2008), aprovechando el marco de los clusters turísticos, realiza un análisis en tres ciudades de Andalucía. Este se centra en los individuos o grupos que operan la actividad turística y las relaciones productivas establecidas entre ellos, revisando la relevancia del capital social para el desarrollo económico local por medio del estudio de los procesos que se presentan en la colaboración entre los actores públicos y privados a través del análisis de las redes sociales. Entre sus hallazgos estála correlación estrecha entre las tres ciudades estudiadas, el nivel de desarrollo turístico y las características de las relaciones de los actores que participan en esta actividad. En este estudio se aprecia que al ser más elevado el desarrollo turístico la densidad también se eleva, así como el número de actores centrales de las redes, la estabilidad y la formalidad existentes en el proceso que se lleva a cabo en turismo. Por lo anterior, el autor señala que el capital social está vinculado con el nivel de desarrollo turístico, lo que repercute en el desarrollo económico de la localidad y de todos los involucrados. 
En México, entre las investigaciones que abordan el capital social de zonas turísticas se encuentran las realizadas acerca de la Reserva de la Biósfera de los Tuxtlas, en Veracruz, y Cerro Prieto, en la Reserva de la Biósfera de la Mariposa Monarca en Michoacán. En la primera investigación, dirigida por Paré y Fuentes (2007), se revisan proyectos y programas de conservación ambiental en la región de los Tuxtlas, donde es requerida la participación comunitaria, y por lo tanto se ha hecho presente la organización de los recursos de la localidad, con lo que el "capital social se ha fortalecido a través de estos proyectos ecoturísticos en los que se ha evidenciado la inclusión de las mujeres en estas actividades, las cuales han desarrollado nuevas habilidades, con lo cual han logrado el acceso a otro tipo de roles diferentes a los tradicionales y se ha valorado su participación en el desarrollo de este tipo de proyectos" (Sandoval y Ortega, 2016, p. 3). En la segunda, que versa sobre la Reserva de la Biosfera de la Mariposa Monarca en Michoacán, realizada por Merino (2004), se observa que la falta de capital social en Cerro Prieto ha ocasionado cierta debilidad en la estructura organizativa al no permitir el acceso a las asambleas ejidales a los no ejidatarios, lo que propicia una falta de integración y de comunicación que frena el desarrollo de la localidad.

En cuanto a los Pueblos Mágicos, los estudios se han dirigido a documentar situaciones de diversa índole evidenciado problemáticas que pudieran ser abordadas para su solución desde la teoría del capital social. En el caso de Tepoztlán, que cuenta con la denominación de Pueblo Mágico y que ha recibido inversión pública para el desarrollo del turismo, los individuos de la localidad externan su inquietud sobre este tema: "los habitantes se sienten ajenos a ello, ya que han mencionado, en entrevistas realizadas a residentes del lugar, que solo se han visto beneficiados ciertos grupos de personas (empresarios) que se dedican al ramo turístico" (Alvarado, Valenzuela, Saldaña y Sorani, 2013, p. 17). Sin embargo, años atrás, cuando Tepoztlán perdió su nombramiento como Pueblo Mágico por no cumplir con algunos criterios de evaluación, se registró una capacidad elevada de organización por parte de los pobladores, con lo cual recuperó el nombramiento (Dávila, 2011). Este poder de organización, de ser potenciado a través de los enfoques del capital social, pudiera generar una gran cantidad de beneficios para la población en general y para todos aquellos que viven de la derrama turística del lugar.

Los pobladores del Pueblo Mágico de San Sebastián del Oeste consideran mínima la retribución de las empresas turísticas a la economía local, ya que los paquetes turísticos suelen contratarse con empresas foráneas, que perciben el beneficio económico de la actividad turística, mientras que la visita en sí produce 
algunos impactos negativos en la localidad, como la "alteración de la tranquilidad del poblado, la contaminación, la aculturación, una separación entre visitantes y pobladores legítimos del lugar" (Baños, Muñoz, y Tovar, 2014, p. 25).

Por otro lado, en Magdalena de Kino, Sonora, "se piensa que los principales beneficiarios del turismo son los comerciantes, los restaurantes, el gobierno y en penúltimo lugar la propia comunidad. Son los servicios ligados al turismo a quienes se percibe como ganadores del flujo de visitantes" (Enríquez, Guillén, Valenzuela y Jaime, 201, p. 83).

En Cuitzeo, Michoacán, como resultado de una consulta a sus pobladores sobre el nombramiento de este como Pueblo Mágico, se observó una tendencia elevada a considerar que el turismo contribuye al desarrollo económico y social de sus habitantes. Sin embargo, al preguntarles acerca de los beneficios que obtienen sus familias por tener Cuitzeo tal distinción, la mitad de los entrevistados refirió que no ha recibido beneficios derivados de tal denominación. Además, una parte de la población cree que la actividad turística en Cuitzeo, ejercida a través del programa Pueblos Mágicos, "es una actividad que sólo beneficia a un pequeño porcentaje de personas, el cual integra a comerciantes y autoridades y que, por tanto, los beneficios del turismo, están lejos de alcanzar a las masas populares de la localidad; provocando con ello, fenómenos de descontento y desvinculación ciudadana” (García y Guerrero, 2014, p. 92).

Como hemos podido observar en los casos relacionados con los Pueblos Mágicos, aunque cada pueblo presente rasgos propios, el común denominador es la falta de involucramiento de la comunidad, y la percepción de que los destinatarios de los beneficios dimanados de la actividad turística son generalmente personas ajenas a la comunidad, comerciantes o empresarios locales y gobierno, por lo cual se ha dejado de lado a los habitantes y sus capacidades. De ello se infiere que si se desea hacer del turismo una actividad de contribución real, elevar niveles de bienestar, aumentar el empleo y optimizar el aprovechamiento de los recursos, es necesario hacer partícipe a toda la sociedad, estimular la confianza y las redes sociales por medio del capital social para que, a través de estas y del involucramiento de todos los actores sociales (gobierno federal, sociedad civil, prestadores de servicios turísticos y comunidad), se acceda a recursos, relaciones, bienes y servicios que permeen en el grupo social de tal manera que la colaboración genere desarrollo económico, social y ambiental, es decir, se logre la sustentabilidad de la actividad turística. 


\section{BASES TEÓRICAS DEL CAPITAL SOCIAL}

El capital social es una base para el impulso de cualquier plan o programa dirigido a las sociedades. Así, es relevante analizarlo a fin de evidenciar que su uso conlleva el aumento de beneficios sociales, mayor desarrollo y una mayor igualdad social. "Lo importante del capital social para los individuos y los grupos que lo poseen es la potencialidad que les confiere y de la que carece un individuo aislado" (Flores y Rello, 2003, p. 3). Por lo que el establecimiento de redes sociales aumenta las oportunidades de quienes las integran mediante la obtención de información oportuna de los planes y proyectos que se implementarán y del establecimiento de metas y objetivos dirigidos a la obtención de beneficios sociales.

A la fecha, el capital social se ha abordado desde varias perspectivas que han sustentado la necesidad de las relaciones sociales como medio de organización para mejorar la calidad de vida de las localidades estudiadas. A continuación se hará un repaso histórico de estas visiones con el fin de reconocer la importancia de las interacciones sociales en el aumento del sentimiento de bienestar social o económico.

Para Hanifan (1916), el capital social "tiene la tendencia a hacer que sustancias tangibles cuenten para la mayoría de las personas en su vida diaria; estas sustancias pueden ser denominadas 'buena voluntad', 'compañerismo', 'simpatía' y 'relaciones sociales' que se dan entre los individuos". Este autor hace hincapié en que "la buena voluntad, el compañerismo, la empatía y las relaciones sociales entre individuos y familias pueden mejorar la calidad de vida en las comunidades" (Hanifan, cit. en Saiz, 2008, p. 251).

Pierre Bourdieu (1985), en The forms of capital, pone de manifiesto que por medio del capital social es posible entender las diferencias que pueden hacerse presentes en la obtención de beneficios si se cuenta con influencias o relaciones. Para Bourdieu, el capital social es "el agregado de los recursos reales o potenciales ligados a la posesión de una red durable de relaciones más o menos institucionalizadas de reconocimiento mutuo" (Bourdieu, 1985, p. 248). El volumen de capital social desarrollado dependerá de las relaciones y conexiones que el grupo posea, $\mathrm{y}$ no de otras formas de capital.

James Samuel Coleman, por su parte, presenta su formulación de capital social en un trabajo publicado en 1988 en la American Journal of Sociology, en el cual introduce la acción social en dos vertientes intelectuales: en la primera caracteriza el trabajo de los sociólogos, y establece que las acciones del actor están gobernadas por normas sociales, reglas y obligaciones; en la segunda caracteriza el trabajo de 
los economistas, y ve que el actor alcanza sus metas movido por interés propio y maximiza su utilidad. Asimismo, el autor menciona algunas relaciones sociales que pueden constituir recursos de capital útiles para los individuos, entre las que se encuentran las obligaciones, las expectativas y las estructuras de confianza, los canales de información, las normas y las sanciones efectivas.

Robert D. Putnam introduce el término de capital social en su libro Making Democracy Work, en el que señala que aspectos de las organizaciones sociales como las redes, las normas y la confianza dan la pauta para la acción y la colaboración para un beneficio común (Putnam, 1993). En este libro, el autor documenta el estudio realizado acerca de los gobiernos regionales de Italia y busca localizar los contextos que permiten el establecimiento de instituciones fuertes, representativas y responsables; además, explica las razones por las que algunos gobiernos (que tienen como forma de organización social la democracia) son exitosos ahí donde otros han fracasado. En cuanto a su aportación al capital social, Putnam confiere un papel relevante a la confianza, las normas de reciprocidad y las redes de compromiso cívico. En su libro más reciente Bowling Alone, Putnam nos muestra cómo la sociedad actual se encuentra cada vez más desconectada de los lazos familiares, vecinales, amistosos y de las estructuras democráticas. A la vez, muestra la forma en que podemos volver a conectarnos para alcanzar de nuevo la unión que eleva la productividad de las sociedades.

Para Ostrom y Ahn (2003, p. 156), el capital social genera la plataforma para "abordar la cuestión de cómo acelerar el desarrollo económico y la gobernabilidad democrática e incorporan a la acción colectiva factores como confianza y normas de reciprocidad, redes y formas de participación civil y reglas o instituciones formales e informales". Estos factores, como hemos podido observar, no son de reciente incorporación; sin embargo, "esta perspectiva le pide a teóricos de la acción colectiva que salgan del mundo del razonamiento puro y aborden cuestiones empíricas de políticas públicas que son cuestiones de importancia práctica” (Ostrom y Ahn, 2003, p. 156).

Para el Banco Mundial (s/f), "el capital social se refiere a las instituciones, relaciones y normas que forman la calidad y la cantidad de interacciones sociales de una sociedad". La unión de las partes que integran a esta sociedad da pie a su prosperidad y desarrollo sostenible. "El capital social no es sólo la suma de las instituciones que sustentan una sociedad, es el pegamento que las mantiene unidas" (Banco Mundial, s/f).

Por otro lado, Javier Díaz Albertini ha hecho un estudio sobre este tema en el que describe el capital social como aquel que "mide la sociabilidad de un conjunto 
humano y aquellos aspectos que permiten que prospere la colaboración y el uso, por parte de los actores individuales, de las oportunidades que surgen en estas relaciones sociales" (Díaz Albertini, 2003, p. 251). Para este autor, es imprescindible un análisis del escenario de la confianza basada en las expectativas y de las redes sociales en los vínculos verticales involucrados, a fin de detectar aquellas relaciones que reporten beneficios a la comunidad, o evidenciar la necesidad de fortalecerlas por parte de los actores gubernamentales, "dado que la distribución y acceso a los recursos que habilitan y promueven la acción colectiva de los recursos recae en el Estado" (Díaz Albertini, 2003, p. 257). Por lo que, para facilitar y fortalecer el capital social, es necesario analizar la confianza y las redes sociales. El estudio de la confianza debe basarse en el examen de las expectativas, porque la confianza mutua es edificada sobre aquello que los individuos o grupos esperan de la actuación de los demás involucrados. Las redes sociales deben estudiarse desde la funcionalidad de las mismas redes como facilitadoras de información, conocimiento y reducción del oportunismo.

Aun cuando se han descrito algunos beneficios que conlleva el uso del capital social, es importante hacer hincapié en las potenciales implicaciones negativas de este en caso de no dirigir las relaciones sociales hacia el logro de metas y objetivos establecidos previamente. A este capital social se le podría llamar capital social negativo, "que distorsionan e incrementan el costo de las transacciones, y reducen las posibilidades de especialización. La guerra, la delincuencia, la corrupción, las amenazas, la discriminación, los actos de discriminación, terrorismo y destrucción del medio ambiente son algunas de sus consecuencias" (Robison, Siles, Schmid, 2003, p. 92).

\section{Capital SOCIAL y LA VERTICALIDAD DE SUS RELACIONES}

La propuesta de Díaz Albertini (2003) se enfoca en el análisis de las relaciones o eslabones verticales entre las organizaciones de base y el resto de la sociedad, mismo enfoque que adopta el presente estudio. La investigación de este autor se dirige a mostrar la imprescindibilidad de la creación y el establecimiento de eslabones que incluyan a la sociedad, que se encuentren basados en la sinergia y la legitimidad para el desarrollo de una sociedad sustentable.

En el área turística en México, como vimos anteriormente, se ha elaborado una serie de planteamientos en el nivel federal que pretenden fortalecer la infraestructura y las capacidades de las comunidades turísticas integrando el concepto de sustentabilidad en cada una de las acciones tomadas. Sin embargo, al momento 
de poner en marcha estos programas, los actores sociales encargados de la implementación afrontan problemas al centrar la mayoría de las acciones sobre la base de los capitales físicos y humanos existentes dejando de lado el capital social con el que se puede establecer relaciones de cooperación y trabajo comunitario en favor del incremento del bienestar común.

Para examinar las relaciones verticales que se establecen y la forma en que permiten el acceso a recursos, relaciones, bienes y servicios, es necesario el análisis de la confianza basada en las expectativas y de las redes sociales existentes en los actores que intervienen en el proceso.

\section{La confianza sobre la base de expectativas}

La confianza se ha considerado como una de las fuentes más relevantes del capital social debido a que sin ella muchas de las actividades que se realizan cotidianamente se verían opacadas por el sentimiento de incertidumbre que surge en los individuos al dejar en otras manos bienes o responsabilidades. Para los economistas, la confianza es vital porque reduce los riesgos generados por las transacciones, así como los costos de estas últimas.

La confiabilidad, sin embargo, es usada para denotar seguridad en los sistemas empleados o aplicados para la mejora continua en empresas, proyectos, programas, entre otros, por lo que el grado de confiabilidad en los programas que aplican los gobiernos para el desarrollo social y económico de comunidades, poblaciones o países puede favorecer o entorpecer su implementación por parte del grupo receptor. Un alto grado de confiabilidad logra establecer al mismo tiempo un alto grado de predictibilidad y de regularidad, lo que permite accionar de acuerdo con las metas establecidas y con la certeza de que los resultados serán los esperados o, al menos, se acercarán en gran medida a ello.

De acuerdo con Barber (cit. en Díaz Albertini, 2013, p. 262), "la confianza tiene como fundamento tres tipos de expectativas: acerca del tipo de orden social deseado, acerca de las competencias y habilidades de los demás y acerca del nivel de cumplimiento de las obligaciones y responsabilidades de nuestras contrapartes". Estos tipos de expectativas los desarrollaremos de manera sucinta a continuación: a) acerca del tipo de orden social deseado, este viene acompañado de las creencias y la ideología que comparte la sociedad en general, las cuales facilitan el entendimiento y la creación de estrategias encaminadas a obtener beneficios comunes; b) en la confianza, las competencias y las habilidades tienen un papel fundamental, ya que de líderes 
o dirigentes expertos y con conocimientos depende la puesta en marcha efectiva de planes y proyectos de acción y, desde luego, el respaldo de la sociedad, y c) el nivel de cumplimiento de las obligaciones y las responsabilidades de las contrapartes es fundamental al momento de estrechar los lazos de cooperación en conjunto, debido a que la percepción del cumplimiento de esta expectativa genera, entre los participantes, un grado tal de compromiso capaz de impulsar las acciones y cumplir con éxito cualquier actividad o tarea encomendada en beneficio de la sociedad en general.

\section{Redes sociales}

Las redes sociales están conformadas por actores sociales de diferentes niveles o estatus que se vinculan entre sí para obtener beneficios comunes, propios de las actividades que han emprendido en conjunto. Estas actividades pueden derivarse de relaciones profesionales, amistosas o de parentesco. En las comunidades es necesario que el capital social existente, en cualquiera de sus modalidades, sea revisado y alimentado constantemente para elevar el nivel de bienestar de la población que permita el logro del desarrollo local de la sociedad.

Estas redes deben ser conformadas para construir puentes y eslabonamientos que garanticen el acceso a los recursos de manera equitativa, a la planeación como ejercicio participativo y a la integración como espacio pluralista. Al hacer referencia a los recursos, el enfoque no solo se dirige a los recursos físicos, naturales o culturales, sino también son contemplados aquellos que "constituyen las capacidades que las personas y los grupos tienen para actuar sobre el mundo y cambiarlo” (Díaz Albertini, 2003, p. 288). En la planeación, como parte fundamental de todo proceso de desarrollo, debe procurarse la participación de la sociedad para tener un conocimiento detallado de los recursos existentes, además de la generación de los planes en que se establezcan los compromisos que cada una de las partes deberá cumplir. Por último, la integración de los actores sociales en espacios pluralistas es un elemento clave para el logro de la participación de la sociedad en los temas relevantes que les competen.

\section{Análisis de TAPIJUlapa como Caso de estudio}

Tapijulapa es una comunidad urbana con gran potencial turístico, perteneciente al municipio de Tacotalpa, Tabasco. Tiene una extensión territorial de 207 kilómetros 
cuadrados, se encuentra a 90 kilómetros de la ciudad de Villahermosa, a orillas de los ríos Oxolotán y Amatán; enclavada en la selva y rodeada de montañas, a 100 metros sobre el nivel del mar. La tenencia del suelo en la Villa Tapijulapa es ejidal en su mayoría; la propiedad privada solo se ubica en la zona centro de la localidad. Su actividad económica se desarrolla por medio de la ganadería; la producción de granos básicos y plátano; el comercio y el turismo.

Los problemas económicos de la población derivan "de la falta de empleo, lo que mantiene una constante migración a otras regiones, por lo que es necesario inducir incentivos económicos a través del sector turístico y agropecuario, generando los empleos en estos sectores que incrementen los ingresos económicos de la población y en consecuencia mejorarán su calidad de vida" (Ecología y Acción Urbana, 2008 , p. 10). Este es el principal motivo, además de la gran variedad de atractivos turísticos, que orilló a las autoridades municipales y estatales en conjunto con los pobladores de Tapijulapa a solicitar el nombramiento de Pueblo Mágico, dado que el gobierno federal comenzó a entregar nombramientos a aquellos pueblos que cumplían con las características establecidas y que deseaban proyectar en el nivel nacional el valor de su localidad a través de la estructuración de paquetes turísticos innovadores y originales dirigidos a los segmentos de mercado interesados en la cultura, las tradiciones, la aventura y el deporte extremo.

Tapijulapa logró el nombramiento como Pueblo Mágico en 2010; sin embargo, con un estudio de observación realizado en esta comunidad, se detectó que, pese al trabajo ahí invertido, existe una falta de coordinación entre los actores sociales involucrados (comunidad, sociedad, empresas, organizaciones institucionales y autoridades de gobierno participantes en el proyecto), que los ha llevado a manifestar varias situaciones que repercuten de modo desfavorable en la obtención de los beneficios esperados.

Algunas situaciones detectadas al momento de la investigación fueron las siguientes: sentimiento de exclusión por parte de los pobladores de los beneficios derivados del programa; los grupos beneficiados necesitan habilidades de organización y la ayuda de personas intermedias facilitadoras en el acceso a las instituciones y a los recursos externos; parte de los beneficios de la implementación de este programa se ha dirigido a receptores privilegiados, es decir, a personas que han tenido una trayectoria política dentro de la comunidad o a sus familiares; disminución de la cooperación entre la comunidad para lograr objetivos comunes, y falta de seguimiento del cumplimiento de los compromisos pactados por las empresas comunitarias en los proyectos financiados por instituciones gubernamentales. 
Por otro lado, la población involucrada en Tapijulapa como Pueblo Mágico está integrada por diferentes actores sociales relacionados con la actividad turística de manera directa o indirecta, a los cuales se les ha clasificado para su correcto estudio en: a) comunidad de Tapijulapa; b) organizaciones de base; c) organizaciones intermedias institucionales, civiles y mixtas, y d) instituciones estatales. A continuación se describen la composición de cada una de ellas.

a) Comunidad de Tapijulapa. Está compuesta por 2846 personas, de las cuales 1390 son hombres y 1456 son mujeres. Su división geopolítica consta de siete colonias: Sabanilla, Colonia Isabel, Nueva Creación, Carretera Oxolotán, La Loma, La Curva y el Centro.

b) Las organizaciones de base. Son aquellas que están integradas por habitantes de Tapijulapa. Cabe aclarar que estas organizaciones conforman dos grupos. El primer grupo está constituido por organizaciones cuyo fin es beneficiar a sus integrantes (cooperativas) o a la población en general (parroquia, grupo protestante y grupo cultural). Mientras que el segundo grupo se organiza para brindar diferentes servicios turísticos al visitante buscando lucrar con esas actividades. Se denominará "organizaciones de base de la localidad" al primer grupo y "prestadores de servicios turísticos” al segundo.

En Tapijulapa existen seis organizaciones de base de la localidad cuyo fin es beneficiar de manera directa o indirecta tanto a los integrantes de la organización misma como a la comunidad en general. Estas organizaciones son las siguientes: un grupo parroquial, un grupo cultural, tres cooperativas (El Sabor de la Sierra, Cadenas Productivas Mutusay, Marca Colectiva Mutusay) y un grupo protestante.

Las organizaciones de prestadores de servicios turísticos están integradas por 16 prestadores de servicios turísticos diferenciados en cuatro tipos: servicios de alimentación, hospedaje, guías de turistas y centros recreativos.

c) Organizaciones intermedias institucionales, civiles y mixtas. Las organizaciones intermedias institucionales están integradas por las instituciones de gobierno que se han visto involucradas de manera directa en el programa de Pueblo Mágico de Tapijulapa a través de proyectos o acciones dirigidas a la mejora de las condiciones sociales, económicas y ambientales de la localidad. De estas instituciones se distinguen, por su participación y apoyo a las actividades dirigidas a estos fines, la Secretaría de Desarrollo Social y Económico, la Comisión Nacional de Derechos Indígenas, el Instituto Estatal de Cultura de Tabasco, el Instituto Nacional de Antropología e Historia y el Instituto para el Fomento de las Artesanías de Tabasco. 
Las organizaciones intermedias civiles están integradas por la parroquia, las universidades, el fondo regional; por líderes de opinión y representantes de partidos políticos.

El Comité de Pueblos Mágicos es una organización mixta configurada por diferentes instituciones de los gobiernos estatal, local y federal, así como por representantes de la localidad con amplio conocimiento de las necesidades y de la trayectoria turística del pueblo. Estos miembros son clasificados de la siguiente manera: a) miembros con voz y voto; b) miembros honorarios con voz, y c) invitados permanentes.

d) Institucionalidad estatal. Está integrada por aquellas instituciones directamente involucradas en la implementación y buen manejo del Pueblo Mágico de Tapijulapa. Se identificaron cuatro dependencias: la Secretaría de Desarrollo Económico y Turismo, el Municipio de Tacotalpa, la Dirección de Fomento Económico y Turismo de Tacotalpa y el delegado de Tapijulapa.

\section{Metodología}

Los fenómenos que se presentan actualmente son de diversa índole y tan complicados que es necesario ver todos los ángulos del problema con la finalidad de entenderlo en profundidad y, con base en los resultados, proponer y realizar acciones que hagan posible su solución. Usar un solo enfoque, cualitativo o cuantitativo, no permite ver la complejidad de la problemática, y se corre el riesgo de dar soluciones a medias; por lo tanto, en esta investigación se empleó un enfoque mixto de diseño exploratorio secuencial realizado en dos etapas, una cualitativa y otra cuantitativa, con un énfasis mayor en la segunda etapa. La primera etapa fue la cualitativa; en esta se recolectó y analizó información mediante el uso de la observación participante y entrevistas semidirectivas. La recolección de datos y el análisis fueron parte de la segunda etapa, a la cual se le dio mayor énfasis usando cuestionarios dirigidos a la cuantificación de la percepción de la población acerca de las variables estudiadas. En total, se aplicaron 237 los cuestionarios en esta etapa.

La investigación proporcionó información diversa para análisis: identificación de la problemática, características de la composición de la población de Tapijulapa como Pueblo Mágico (descrita en la sección anterior), cuantificación de la percepción de la comunidad sobre las relaciones verticales, y los vínculos entre la comunidad y los diferentes actores sociales involucrados en el proceso. 


\section{Construcción del indice de capital social \\ Como se mencionó anteriormente, para examinar las relaciones verticales y la forma en que estas permiten el acceso a los recursos, las relaciones y los bienes y servicios se analizó la confianza basada en las expectativas y de las redes sociales en la comunidad mediante un cuestionario. Las preguntas aplicadas en el cuestionario fueron las siguientes.}

\section{CUESTIONARIO PARA MEDIR EL CAPITAL SOCIAL EN LA COMUNIDAD DE TAPIJULAPA*}

\begin{tabular}{|c|c|}
\hline Confianza & Redes sociales \\
\hline Creencias compartidas e ideología & Acceso a recursos \\
\hline $\begin{array}{l}\text { 1. Todos los miembros de la comunidad de Tapijulapa } \\
\text { son dignos de confianza. }\end{array}$ & $\begin{array}{l}\text { 1. El Comité de Pueblo Mágico dirige sus acciones a la } \\
\text { comunidad de Tapijulapa de acuerdo con las necesida- } \\
\text { des detectadas en las interacciones que tiene con los } \\
\text { habitantes. }\end{array}$ \\
\hline 2. Es fácil confiar en la ayuda de extraños. & $\begin{array}{l}\text { 2. Las relaciones existentes entre los organismos guber- } \\
\text { namentales locales y estatales con la comunidad genera } \\
\text { que las acciones que se dirigen a Tapijulapa se realicen de } \\
\text { acuerdo con las necesidades reales de los habitantes. }\end{array}$ \\
\hline $\begin{array}{l}\text { 3. El nivel de confianza entre los miembros de la } \\
\text { comunidad ha aumentado a raíz del nombramiento de } \\
\text { Tapijulapa como Pueblo Mágico. }\end{array}$ & $\begin{array}{l}\text { 3. Las diferentes asociaciones civiles promueven el } \\
\text { bienestar y la mejora de los habitantes de Tapijulapa por } \\
\text { medio de las relaciones que sostienen con diferentes } \\
\text { organizaciones gubernamentales. }\end{array}$ \\
\hline $\begin{array}{l}\text { 4. Confío en el Comité de Pueblos Mágicos como } \\
\text { intermediario entre los niveles de gobierno y la población } \\
\text { de la localidad. }\end{array}$ & $\begin{array}{l}\text { 4. La Secretaría de Desarrollo Económico y de Turismo } \\
\text { trabaja en conjunto con los empresarios turísticos, la } \\
\text { academia y la comunidad para lograr el desarrollo de Ta- } \\
\text { pijulapa como destino turístico y así mejorar la condición } \\
\text { de vida de sus habitantes. }\end{array}$ \\
\hline $\begin{array}{l}\text { 5. Confío en que las organizaciones gubernamentales } \\
\text { locales y estatales buscan el bien común de los habitantes } \\
\text { de Tapijulapa como Pueblo Mágico. }\end{array}$ & $\begin{array}{l}\text { 5. Si participo, trabajo y apoyo las iniciativas promovi- } \\
\text { das por el Comité de Pueblo Mágico, la Secretaría de } \\
\text { Desarrollo Económico y de Turismo y organizaciones } \\
\text { gubernamentales locales y estatales, es más probable que } \\
\text { me entere de las formas en que puedo acceder a los bene- } \\
\text { ficios que trae consigo el nombramiento de Tapijulapa } \\
\text { como Pueblo Mágico. }\end{array}$ \\
\hline $\begin{array}{l}\text { 6. El trabajo realizado por el Comité de Pueblos Mági- } \\
\text { cos, la Secretaría de Desarrollo Económico y de Turismo, } \\
\text { las organizaciones gubernamentales locales y estatales } \\
\text { me hace confiar en que los cambios que se generan en } \\
\text { Tapijulapa serán positivos. }\end{array}$ & $\begin{array}{l}\text { 6. Si participo, trabajo y apoyo las actividades dirigidas } \\
\text { por la sociedad civil (universidades, asociaciones de } \\
\text { empresarios y partidos políticos), es más probable que } \\
\text { obtenga a cambio información, conocimientos y capa- } \\
\text { citaciones que podrían ser útiles para mejorar mi trabajo } \\
\text { cotidiano y fuentes de ingresos. }\end{array}$ \\
\hline
\end{tabular}


CUESTIONARIO PARA MEDIR EL CAPITAL SOCIAL

EN LA COMUNIDAD DE TAPIJULAPA*

(continuación)

\begin{tabular}{l} 
Confianza \\
\hline \multicolumn{1}{c}{ Creencias compartidas e ideología } \\
\hline 7. Considero que los proyectos promovidos por el \\
Comité de Pueblos Mágicos, así como por las organiza- \\
ciones gubernamentales locales y estatales, han buscado \\
cambiar equitativamente las condiciones sociales y \\
económicas de la comunidad.
\end{tabular}

8. Los proyectos implementados en Tapijulapa han logrado que la población se encuentre más satisfechay más integrada.

Redes sociales

Acceso a recursos

\section{Capacidad de planeación}

7. El Comité de Pueblos Mágicos, la Secretaría de

Desarrollo Económico y de Turismo, así como las organizaciones gubernamentales locales y estatales propician la participación de la comunidad en la creación de estrategias y solución de problemas ambientales, de desarrollo urbano y de turismo.

9. Considero que los programas implementados en Tapijulapa como Pueblo Mágico motivan la participación de las mujeres en las actividades remuneradas.

8. El Comité de Pueblos Mágicos y la Secretaría de Desarrollo Económico y de Turismo se esfuerzan por conocer los recursos con los que cuenta la comunidad y cómo pueden ser complementados por otras organizaciones (civiles o gubernamentales).

10. Estoy dispuesto a generar ideas o apoyar las ideas de los grupos que busquen la transformación que lleve al progreso de la comunidad.

9. El Comité de Pueblos Mágicos, la Secretaría de Desarrollo Económico y de Turismo y las organizaciones gubernamentales locales y estatales se esfuerzan en empoderar (fortaleciendo los conocimientos) a la comunidad para que dé seguimiento y evalúe los planes y proyectos que se llevarán a cabo.

11. El Comité de Pueblos Mágicos se esfuerza por lograr, para la comunidad, los niveles de bienestar alcanzados en Tequila, Jalisco, o en otros Pueblos Mágicos exitosos.

10. La participación en las iniciativas de las diferentes organizaciones se ve condicionada por la confianza que tenga hacia el Comité de Pueblos Mágicos, así como a las organizaciones gubernamentales locales y estatales.

12. El tipo de turismo que recibe Tapijulapa como Pueblo Mágico realmente deja los beneficios esperados por la sociedad de Tapijulapa.

\section{Integración como espacios pluralistas}

13. Los principales promotores, internos y externos, de Tapijulapa como Pueblo Mágico enfocan sus actividades y programas en la atracción del tipo de turistas que la sociedad de Tapijulapa necesita.

11. El Comité de Pueblos Mágicos, la Secretaría de Desarrollo Económico y de Turismo y la sociedad civil (universidades, asociaciones de empresarios y grupos políticos) crean formas de obtener proyectos y recursos que integren a la sociedad sin importar edad, raza, sexo, estado civil u otros.

14. Conozco los beneficios sociales, económicos y ambientales que podrían derivar del nombramiento de Tapijulapa como Pueblo Mágico.
12. El Comité de Pueblos Mágicos y la Secretaría de Desarrollo Económico y de Turismo buscan maneras de establecer acuerdos y de solucionar conflictos para integrar las iniciativas de los miembros de la comunidad.

13. Como ciudadano, siento que mis ideas o aportaciones son tomadas en cuenta. 
CUESTIONARIO PARA MEDIR EL CAPITAL SOCIAL

EN LA COMUNIDAD DE TAPIJULAPA*

(continuación)

\begin{tabular}{ll}
\hline \multicolumn{1}{c}{ Confianza } & \multicolumn{1}{c}{ Redes sociales } \\
\hline \multicolumn{1}{c}{ Creencias compartidas e ideología } & \multicolumn{1}{c}{ Acceso a recursos } \\
\hline $\begin{array}{l}\text { 15. El Comité de Pueblos Mágicos cuenta con las habili- } \\
\text { dades, competencias y destrezas necesarias para lograr el } \\
\text { bienestar deseado por los habitantes de la comunidad. }\end{array}$ & $\begin{array}{l}\text { 14. El Comité de Pueblos Mágicos, la Secretaría de De- } \\
\text { sarrollo Económico y de Turismo o las organizaciones } \\
\text { civiles (universidades, asociaciones de empresarios y gru- } \\
\text { pos políticos) procuran espacios de encuentro, diálogo, } \\
\text { debate entre diversos sectores de la ciudadanía. }\end{array}$ \\
\hline $\begin{array}{l}\text { 16. Las organizaciones gubernamentales locales y } \\
\text { estatales cuentan con los conocimientos y habilidades } \\
\text { necesarios para lograr los cambios y la transformación } \\
\text { que necesita la comunidad para mejorar sus condiciones } \\
\text { de vida. }\end{array}$ & $\begin{array}{l}\text { 15. Existen relaciones estrechas entre las organizaciones } \\
\text { gubernamentales, el Comité de Pueblos Mágicos, } \\
\text { la sociedad civil y las organizaciones existentes en la }\end{array}$ \\
\hline $\begin{array}{l}\text { 17. El comité de Pueblos Mágicos y las organizaciones } \\
\text { gubernamentales locales y estatales se orientan a la } \\
\text { búsqueda y aplicación de proyectos que lleven beneficios } \\
\text { sociales (con equidad para la población) para mejorar las } \\
\text { condiciones de vida de la comunidad. }\end{array}$ & $\begin{array}{l}\text { 16. Se celebran reuniones en las que se generan consensos } \\
\text { estatal. }\end{array}$ \\
\hline
\end{tabular}

18. El Comité de Pueblos Mágicos y las organizaciones gubernamentales locales y estatales buscan aplicar proyectos turísticos enfocados al cuidado de los recursos ambientales para garantizar su permanencia en el tiempo.

\section{Nivel de cumplimiento de obligaciones}

19. Los miembros del Comité de Pueblos Mágicos evitan tomar ventaja de los cargos conferidos y velan por el bien de la comunidad.

20. Los miembros de las organizaciones gubernamentales locales y estatales evitan actuar de manera oportunistay velan por el bien de la comunidad.

21. Las organizaciones gubernamentales directamente involucradas en Tapijulapa como Pueblo Mágico desarrollan sus actividades con respeto y equidad hacia la población.

22. La mayoría de los integrantes de la comunidad colabora con gusto en actividades dirigidas a lograr el bienestar común de Tapijulapa.

*El presente cuestionario también ha sido utilizado para realizar un análisis factorial usando la técnica de componentes principales. Esos resultados han sido publicados en un artículo previo titulado "El capital social como factor para el desarrollo sostenible en el pueblo mágico de Tapijulapa", El Periplo Sustentable, 31. 
Como es bien conocido, para el capital social no ha sido posible establecer un proceso sistémico que permita medirlo de forma estandarizada en todas las sociedades. Tal situación se puede tomar como una ventaja, debido a que es posible optar por establecer un método de acuerdo con aquellas características que se desean estudiar incorporando niveles y unidades de análisis. Sin embargo, no es posible obviar la dificultad de realizar una estimación directa del capital social.

Para esta investigación, el análisis se realizó a través de un índice de capital dirigido a la comunidad de Tapijulapa, integrado por la relación de los subíndices de confianza y de redes sociales a fin de proporcionar un espectro más amplio de la situación del capital social en esta comunidad. Este índice se formó con objeto de construir indicadores que den cuenta de las relaciones de verticalidad entre la comunidad y los demás actores sociales en Tapijulapa como Pueblo Mágico. La metodología empleada consistió en una ponderación simple, similar a la empleada por el Programa de las Naciones Unidas para el Desarrollo para la estimación del índice de desarrollo humano, solo que, al no existir indicadores previos con los que se pueda realizar una comparación, los subíndices han tomado valores entre 1 y 5 . Estos son los valores asignados a las opciones de respuesta de acuerdo con la escala de Likert para medir percepciones ( $1=$ totalmente en desacuerdo, $2=$ en desacuerdo, $3=$ ni de acuerdo ni en desacuerdo, $4=$ de acuerdo y $5=$ totalmente de acuerdo), empleado en el cuestionario aplicado a la comunidad en general. Un valor de 5 indicaría niveles muy altos de confianza en las redes sociales, lo que estaría propiciando las condiciones óptimas de capital social de la localidad para el proceso de cambio y desarrollo sustentable de Tapijulapa; mientras que un valor de 1 indicaría lo contrario, es decir, un nivel muy alto de desconfianza o, en su caso, deficiencia en las redes sociales; en otras palabras, que el stock de capital social existente no es suficiente para promover los cambios necesarios y el desarrollo sustentable de Tapijulapa.

A continuación se presenta la estructura utilizada en el análisis de la comunidad, a través de los subíndices de confianza, redes sociales y del índice de capital social para la comunidad. Las variables estudiadas, así como sus respectivos indicadores (en los subíndices e índice), tendrán la misma relevancia, debido a que Díaz Albertini (2003), al examinar las relaciones verticales en la facilitación y fortalecimiento de los procesos conducentes a la construcción del capital social, no da un valor específico a cada una de las variables o indicadores estudiados, por lo que se infiere que tienen un tratamiento igual.

El subíndice confianza está conformado por la variable del mismo nombre, e integra los resultados de los siguientes indicadores: 
CCEI $=$ Creencias compartidas e ideología.

$\mathrm{HCD}=$ Habilidades, competencias y destrezas.

$\mathrm{NCO}=$ Nivel de cumplimiento de obligaciones.

Para estimar este subíndice se consideraron los resultados de las respuestas obtenidas en los cuestionarios. La confianza (CON) es el promedio simple de los indicadores ya transformados y representados de la siguiente manera:

$$
\mathrm{CON}=\left(1 / 3^{*} \mathrm{CCEI}+1 / 3^{*} \mathrm{HCD}+1 / 3^{*} \mathrm{NCO}\right)
$$

En el cuadro 1 se sintetizan las descripciones de los valores en la confianza que presentan entre 1 y 5 .

CuAdro 1. RANGO DE ANÁLISIS PARA EL SUbíNDICE DE CONFIANZA

\begin{tabular}{ll}
\hline \multicolumn{1}{c}{ Rango } & \multicolumn{1}{c}{ Confianza } \\
\hline De 1 a 1.4 & Desarrollo \\
\hline De 1.5 a 1.9 & Nivel muy alto de desconfianza \\
\hline De 2 a 2.4 & Nivel intermede desconfianza de desconfianza \\
\hline De 2.5 a 2.9 & Nivel bajo de desconfianza \\
\hline 3 & Neutro \\
\hline De 3.1 a 3.5 & Nivel bajo de confianza \\
\hline De 3.6 a 4 & Nivel intermedio de confianza \\
\hline De 4.1 a 4.5 & Nivel alto de confianza \\
\hline De 4.6 a 5 & Nivel muy alto de confianza \\
\hline
\end{tabular}

El subíndice de redes sociales está conformado por la variable del mismo nombre, e integra los resultados de los siguientes indicadores:

$\mathrm{AR}=$ Acceso a recursos.

$\mathrm{CP}=$ Capacidad de planeación.

ICEP= Integración como espacios pluralistas.

Para hacer la estimación adecuada de este subíndice se consideraron las respuestas obtenidas en los cuestionarios. Las redes sociales (REDSC) es el promedio simple de los indicadores ya transformados y representados de la siguiente manera: 


$$
\operatorname{REDSC}=\left(1 / 3^{*} \mathrm{AR}+1 / 3^{*} \mathrm{CP}+1 / 3^{*} \mathrm{ICEP}\right)
$$

En el cuadro 2 se encuentra las descripción de los valores en las redes sociales que presentan entre 1 y 5 .

CUAdro 2. RANGO DE ANÁLISIS PARA EL SUbÍNDICE DE REDES SOCIALES

\begin{tabular}{ll}
\hline \multicolumn{1}{c}{ Rango } & \multicolumn{1}{c}{ Redes sociales } \\
\hline De 1 a 1.4 & Desarrollo \\
\hline De 1.5 a 1.9 & Nivel muy alto de deficiencia en las redes sociales \\
\hline De 2 a 2.4 & Nivel intermede deficiencia en le def rederiencia en las reciales \\
\hline De 2.5 a 2.9 & Nivel bajo de deficiencia en las redes sociales \\
\hline 3 & Neutro \\
\hline De 3.1 a 3.5 & Nivel bajo de las redes sociales \\
\hline De 3.6 a 4 & Nivel intermedio de las redes sociales \\
\hline De 4.1 a 4.5 & Nivel alto de las redes sociales \\
\hline De 4.6 a 5 & Nivel muy alto de las redes sociales \\
\hline
\end{tabular}

El índice de capital social para la comunidad de Tapijulapa como Pueblo Mágico se encuentra integrado por dos subíndices: confianza $(\mathrm{CON})$ y redes sociales (REDSC).

$$
\operatorname{CSTPM}=\left(1 / 2^{*} \mathrm{CON}+1 / 2^{*} \mathrm{REDSC}\right)
$$

Los resultados así obtenidos dan la pauta para continuar midiendo el progreso y los avances, o que se presentan en Tapijulapa a raíz de las acciones que se implementen para lograr el desarrollo sustentable de esta comunidad como Pueblo Mágico.

En el cuadro 3 se enuncian las descripciones de los valores para el índice de capital social que presentan entre 1 y 5 .

CUADRO 3. RANGO DE ANÁLISIS PARA EL ÍNDICE DE CAPITAL SOCIAL

\begin{tabular}{ll}
\hline \multicolumn{1}{c}{ Rango } & \multicolumn{1}{c}{ Desarrollo } \\
\hline De 1 a 1.4 & Nivel muy alto de deficiencia de capital social \\
\hline De 1.5 a 1.9 & Nivel alto de deficiencia en el capital social \\
\hline De 2 a 2.4 & Nivel intermedio de deficiencia en el capital social \\
\hline
\end{tabular}




\begin{tabular}{ll}
\hline \multicolumn{1}{c}{ Rango } \\
\hline De 2.5 a 2.9 & Nivel bajo de deficiencia en el capital social \\
\hline 3 & Neutro \\
\hline De 3.1 a 3.5 & Nivel bajo de eficiencia en el capital social \\
\hline De 3.6 a 4 & Nivel intermedio de eficiencia del capital social \\
\hline De 4.1 a 4.5 & Nivel alto de eficiencia del capital social \\
\hline De 4.6 a 5 & Nivel muy alto de eficiencia del capital social \\
\hline
\end{tabular}

\section{Resultados}

En este apartado se exponen los resultados obtenidos en la investigación en la comunidad de Tapijulapa considerada como un análisis de las relaciones verticales que conducen a la construcción del capital social.

1. Confianza basada en las expectativas

CuAdro 4. CONFIANZA BASADA EN EXPECTATIVAS

\begin{tabular}{lcc}
\hline \multicolumn{1}{c}{ Indicadores } & Resultado & Subíndice \\
\hline Creencias compartidas e ideología & 2.9 & 3.0 \\
\cline { 1 - 2 } Habilidades, competencias y destrezas & 3.1 & \\
\hline Nivel de cumplimiento de obligaciones & 2.9 & \\
\hline
\end{tabular}

Fuente: Elaboración propia con datos obtenidos en los cuestionarios aplicados en la comunidad.

En lo que se refiere a la variable confianza en la comunidad, da como resultado 3.0. Esto significa que al evaluar en la comunidad la percepción de ella misma y de las organizaciones intermedias institucionales, mixtas e institucionalidad estatal, en promedio los tres indicadores evaluados arrojan un nivel de confianza neutro, lo que nos lleva a inferir un nivel bajo de desconfianza. Una de las debilidades es aquella relacionada con las creencias e ideologías existentes (primer indicador), debido a que estas no son compartidas por todos los habitantes, lo que ocasiona dificultades de comunicación y, desde luego, obstaculiza la acción de las personas al momento de desempeñar actividades derivadas del nombramiento de Pueblo Mágico. 
Las habilidades, competencias y destrezas (segundo indicador) de las organizaciones gubernamentales locales y estatales, así como del Comité de Pueblos Mágicos, muestran un nivel bajo de confianza, lo que nos lleva a inferir que los conocimientos técnicos y las habilidades de sus dirigentes son poco apreciados por la comunidad. Recordemos que estos son necesarios para lograr la transformación y el desarrollo sustentable que la localidad requiere, porque "si de parte de la comunidad no existe confianza en las competencias, el cambio se convierte en un riesgo demasiado grande" (Díaz Albertini, 2003, p. 267).

El cumplimiento de obligaciones (tercera dimensión) alcanzó un nivel bajo de desconfianza. Con este resultado inferimos, de acuerdo con la percepción de la comunidad, que a los actores encargados de la implementación de la política pública de Pueblos Mágicos les falta compromiso cívico que motive la realización de los planes y programas establecidos con mayor sensibilidad evitando tomar ventaja de la situación y velando por el bien de la comunidad. La confianza es vital en la generación de relaciones que conduzcan al trabajo en conjunto mutuamente beneficioso, ya que "esta es una actitud que se basa en el comportamiento que se espera de la otra persona que participa en la relación que se establece entre ambas” (Durston, 2002, p. 16).

2. Redes sociales

CuAdro 5. Redes SOCIALES

\begin{tabular}{lcc}
\hline \multicolumn{1}{c}{ Indicadores } & Resultado & Subíndice \\
\hline Acceso a recursos & 3.2 & 3.0 \\
\cline { 1 - 2 } Capacidad de planeación & 3.0 & \\
\cline { 1 - 2 } Integración como espacios pluralistas & 2.8 & \\
\hline
\end{tabular}

Fuente: Elaboración propia con datos obtenidos en los cuestionarios aplicados en la comunidad.

El subíndice de redes sociales para la comunidad da como resultado neutro. Este resultado permite inferir que al ser evaluada en la comunidad la percepción del Comité de Pueblos Mágicos, las organizaciones intermedias institucionales, la sociedad civil y la institucionalidad estatal, predomina la indiferencia, equivalente a un valor neutro. El primer indicador, acceso a los recursos, arroja un nivel bajo en las redes sociales, lo que muestra que las acciones emprendidas por los encargados de dirigir las acciones de desarrollo hacia la comunidad toman levemente en 
cuenta las necesidades reales de la población al dirigir los recursos para mejorar las condiciones de vida de Tapijulapa.

El tema de los recursos es de gran relevancia porque de estos depende "el proceso de composición de un modo de vida, quizás sobre todo en un contexto en donde el sustento de las personas pasa de depender directamente de los recursos naturales, a otro basado en una gama de bienes, fuentes de ingresos, de productos y mercados de trabajo" (Bebbington, 1999, p. 4).

La capacidad de planeación, segundo indicador evaluado, se ubica en un nivel neutro, es decir, la respuesta en cuanto a la percepción positiva y negativa de la capacidad de planeación se compensa para dar este resultado.

El análisis de la percepción de la integración como espacios pluralistas, tercer indicador evaluado, arroja un nivel bajo de deficiencia en las redes sociales, lo que lleva a inferir una ligera fragilidad en este ámbito que, de no tomar acciones para elevar el sentimiento de participación, continuaría propiciando un sentimiento de inconformidad en los pobladores, con lo cual disminuirá su participación, ya que "la fragmentación y segmentación social existentes en muchos de nuestros países son el resultado de la ausencia de mecanismos integradores, sean estos desde las bases o desde la llamada sociedad mayor" (Díaz Albertini, 2003, p. 290).

3. Índice de capital social para la comunidad

TABLA 6. ÍNDICE DE CAPITAL SOCIAL

\begin{tabular}{llll}
\hline & Variables & Subíndice & Índice \\
\hline Confianza & 3.0 & \multirow{2}{*}{3.0} & \\
\cline { 1 - 2 } Redes sociales & 3.0 & & \\
\hline
\end{tabular}

Fuente: Elaboración propia con base en los datos obtenidos en el cuestionario aplicado en la comunidad.

Para la comunidad, el índice de capital social da como resultado 3.0, después de evaluar la percepción de las organizaciones intermedias institucionales, organizaciones mixtas, institucionalidad estatal y ellas mismas con respecto de la confianza y de las redes y el acceso a oportunidades como fuentes de capital social. Este resultado permite inferir que a pesar de que la confianza (primera variable estudiada) es una de las fuentes de capital social que facilitan la cooperación voluntaria, el proceso de cambio y desarrollo sustentable no alcanza a ser parte fundamental de las relaciones diarias que se entretejen entre los actores sociales involucrados en el proceso. 
El estudio de las redes sociales, segunda variable estudiada, arroja un resultado neutro, por lo cual se infiere que las relaciones sociales que se entretejen en la redes existentes no logran ser lo suficientemente fuertes para generar vínculos que propicien una distribución de recursos equilibrados y de acuerdo a las necesidades de la población, una planeación como ejercicio participativo y la integración como espacios pluralistas. Estas acciones que, según la teoría de Díaz Albertini (2003), son primordiales para el establecimiento de una serie de eslabonamientos y para el acceso a oportunidades, deberán fortalecerse a fin de que Tapijulapa alcance su desarrollo sustentable como Pueblo Mágico.

\section{Conclusiones}

Es claro que el programa Pueblos Mágicos ha propiciado cierto grado de desarrollo social y económico de la población a través de programas dirigidos a aumentar y mejorar los servicios turísticos de la localidad. Sin embargo, el capital social no ha sido contemplado aún como herramienta que dirija los esfuerzos de los habitantes de la localidad y de los actores sociales involucrados hacia el desarrollo sustentable de Tapijulapa a raíz de la implementación de esta política turística.

La confianza basada en las expectativas, aunque no ha mostrado situaciones negativas en el proceso, tampoco lo han sido positivas. Con esto, se demuestra que no alcanza a ser un factor que impulse el desarrollo social, económico y ambiental de Tapijulapa. Ello, tomando en cuenta que: A) Las creencias compartidas e ideología, primera indicador evaluado, no son las mismas para todos los habitantes de la localidad, lo que ocasiona dificultades para el establecimiento de una comunicación efectiva y para la integración de los actores sociales locales. Este escenario no favorece el desarrollo exitoso de las actividades derivadas de este nombramiento. B) En cuanto al indicador siguiente, habilidades, competencias y destrezas, las capacidades de los actores encargados de la implementación no son lo suficientemente valoradas por los habitantes de Tapijulapa, lo cual podría estar frenando la cooperación de estos últimos. C) Respecto del último indicador evaluado, el nivel de cumplimiento de obligaciones, se registra una leve deficiencia en cuanto al compromiso cívico por parte de las autoridades encargadas de este proceso, que les impide, de acuerdo con la percepción registrada, realizar los planes y programas con mayor sensibilidad y procurando que los beneficios se vean reflejados por igual en toda la comunidad. 
Para las redes sociales, el escenario no difiere mucho del anterior; este muestra una falta de orientación hacia el uso eficiente de los eslabones existentes que facilite, para los integrantes de la comunidad, el acceso a los beneficios derivados de este nombramiento. Ello, tomando en cuenta que: A) Para el acceso a recursos, primer indicador evaluado, las acciones emprendidas por los encargados de la implementación no han dado prioridad a las necesidades reales de la población y de la localidad en general. B) En lo que se refiere al siguiente indicador evaluado, la capacidad de planeación, ha sido mínimo el involucramiento de la sociedad para la creación de estrategias y solución de problemas, por lo cual se han desaprovechado en gran medida los conocimientos de los locales sobre el territorio y la cultura. C) Respecto del último indicador evaluado, la integración como espacios pluralistas, se ha registrado una pequeña deficiencia, ya que los evaluados consideran que no existen espacios suficientes donde puedan ser escuchados, lo cual limita la interacción entre la comunidad y los demás actores sociales.

De acuerdo con Díaz Albertini (2013), para enriquecer el capital social es indispensable comprender la relación del grupo de intelectuales y técnicos con aquellas comunidades o sociedades excluidas, porque estas facilitan las actividades colectivas principalmente. Esta es la razón por la que la confianza deberá construirse como plataforma de impulso de relaciones sociales, que permitan la creación de ideologías apropiadas y de acuerdo con la situación social, económica y ambiental del grupo social. La confianza, al estar bien cimentada, dará paso a un alto compromiso cívico tanto de las autoridades involucradas como de cada uno de los actores sociales que integran la comunidad, con lo que se disminuirán las intervenciones oportunistas y se establecerán relaciones con un alto sentido de responsabilidad y deseos de velar por el bien de la comunidad.

Las redes tienen un papel muy importante al momento de realizar trabajo en conjunto, principalmente para generar puentes y eslabonamientos que hagan posible el acceso a los recursos y a las oportunidades. Tal acceso suele ser un problema para aquellas personas que no tienen ocasión de poseer bienes y oportunidades, por lo que los actores sociales directamente involucrados en la implementación del programa Pueblos Mágicos deben establecer estrategias que posibiliten la integración de todos los sectores de la sociedad de Tapijulapa. Con esta integración, además de generar planes y proyectos de acuerdo con las necesidades reales de la comunidad, las personas podrán obtener recursos provenientes de los grupos en los que se encuentran inmersos, tales como los recursos que conlleva la participación, la movilización y los materiales o bienes comunes de la comunidad. 


\section{BibLIOgRAFÍA}

Alfaro, J., y Zambrano, A. (2009). Psicología comunitaria y políticas sociales en Chile. Psicologia \& Sociedade, 21(2): 275-282. Recuperado de http://www.scielo.br/pdf/ psoc/v2ln2/v21n2a15.pdf.

Alvarado, C.; Valenzuela, A.; Saldaña, M. C., y Sorani V. (2013). Pueblo mágico y la conservación del patrimonio. Caso de estudio Tepoztlán, Morelos (2002-20012). Topofilia. Revista de Arquitectura, Urbanismo y Ciencias Sociales, IV(3): 1-20. Recuperado de http://148.228.173.140/topofiliaNew/assets/1-m5c-car-ava-msfvsd.pdf.

Banco Mundial(s/f).SocialCapital. The World Bank Group. Recuperadodehttp://web.worldbank.org/WBSITE/EXTERNAL/TOPICS/EXTSOCIALDEVELOPMENT/EX TTSOCIALCAPITAL/0,,contentMDK:20187568 menuPK:410388 pagePK:1 48956 piPK:216618 theSitePK:401015,00.html.

Baños, J.A.; Muñoz, M., y Tovar, R. (2013). El Pueblo Mágico de San Sebastián del Oeste, Jalisco. Topofilia. Revista de Arquitectura, Urbanismo y Ciencias Sociales, IV(1): 1-33. Recuperado de http://148.228.173.140/topofiliaNew/assets/8-vvn1.pdf.

Bebbington, A. (1999). Capitals and Capabilities: A Framework for Analyzing Peasant Viability, Rural Livelihoods and Poverty. World Development, 27(12): 2021-2034. DOI: http://dx.doi.org/10.1016/S0305-750X(99)00104-7.

Bourdieu, P. (1985). The Forms of Capital. En J. Richardson (ed.). Handbook of Theory and Research for the Sociology of Education. Nueva York, Estados Unidos: Greenwood.

Coleman, J. (1988). Social Capital in the Creation of Human Capital. The American Journal of Sociology, 94. Supplement: Organizations and Institutions: Sociological and Economic Approaches to the Analysis of Social Structure, S95-S120. Recuperado de http://faculty.washington.edu/matsueda/courses/587/readings/ Coleman\%201988.pdf.

Cruz, M. A.; Rodríguez, A., y Zamora, P. M. (2013). Pueblos Mágicos: ¿Proyecto de revaloración e inserción dentro del mercado turístico nacional? Tapalpa y Mazamitla. Topofilia. Revista de Arquitectura, Urbanismo y Ciencias Sociales, IV(1).

Díaz Albertini, J. (2003). Capital social, organizaciones de base y el Estado: Recuperando los eslabones perdidos de la sociabilidad. En R. Atria y M. Siles (eds.). Capital social y reducción de la pobreza en América Latina y el Caribe: En busca de un nuevo paradigma. Santiago, Chile: Comisión Económica Para América Latina y el Caribe. 
Durston, J. (2000). ¿Qué es el capital social comunitario? Santiago, Chile: Comisión Económica Para América Latina y el Caribe, División de Desarrollo Social (Serie Políticas Sociales, 38).

Durston, J. (2002). El capital social campesino en la gestión del desarrollo rural. Díadas, equipos, puentes y escaleras. Santiago, Chile: Comisión Económica para América Latina y el Caribe.

EnRíluez, J.; Guillén, M.; Valenzuela, B., y Jaime M. E. (2015). Magdalena de Kino, Sonora. Percepciones de la población con respecto al patrimonio cultural y el turismo. Topofilia. revista de Arquitectura, Urbanismo y Territorios, V(1): 67-88. Recuperado de http://148.228.173.140/topofiliaNew//assets/enriquez-et-al.pdf. Flores, M., y Rello, F. (2003). Capital social: Virtudes y limitaciones. En R. Atria y M. Siles (comps.). Capital social y reducción de la pobreza en América Latina y el Caribe: En busca de un nuevo paradigma. Santiago, Chile: Comisión Económica para América Latina y el Caribe.

Fondo Nacional de Fomento al Turismo (2008). Programa de desarrollo turístico, Tapijulapa, Tabasco. Ecologia y Acción Urbana. Villahermosa, Tabasco, México: Fondo Nacional de Fomento al Turismo.

GaLÁn, J. L., y Castro, I. (2004). Las relaciones interorganizativas como fuente de capital social. Universia Business Review (2): 104-117. Recuperado de http://www.redalyc. org/articulo.oa?id=43300208.

Galaso, P. (2005). Capital social y desarrollo económico. Los casos de Silicon Valley y Villa el Salvador. Revista de Ciencias Sociales y Humanidades, 15(27): 161-188. Recuperado de: http://www.redalyc.org/articulo.oa?id=85902707.

García, D., y Guerrero García Rojas, H. R. (2014). El programa Pueblos Mágicos: Análisis de los resultados de una consulta local ciudadana. El caso de Cuitzeo, Michoacán, México. Economía y Sociedad, XVIII(31): 71-94.

GRIs, P. (2009). Diferencias en la implementación: Una politica, dos resultados. El caso del Programa Pueblos Mágicos (tesina). Centro de Investigación y Docencia Económicas. Distrito Federal, México.

Hanifan, L. (1916). The Rural School Community Center. Annals of the American Academy of Political and Social Science, 67 (1916): 130-138.

Lew Is, D., y Andrew W. (1985). Trust as a Social Reality. Social Forces, 63(4): 967-985. DOI: $10.2307 / 2578601$.

Loredo, J. L. (2012). Pueblos Mágicos entre el simulacro y la realidad. Topofilia. Revista de Arquitectura, Urbanismo y Ciencias Sociales, III(1). Recuperado de http://148.228.173.140/topofiliaNew/assets/tres3jlloredo.pdf. 
Luyando, R.; Sandoval, L., y Ortega, E. (2016). El capital social como factor para el desarrollo sostenible en el pueblo mágico de Tapijulapa. El Periplo Sustentable (31). Recuperado de https://rperiplo.uaemex.mx/article/view/4886.

Macbeth, J.; Carson, D. y Northcote, J. (2004). Social Capital, Tourism and Regional Development: SPCC as a Basis for Innovation and Sustainability. Current Issues in Tourism, 7(6): 502-522.

Merinero Rodríguez, R. (2008). Micro-cluster turísticos: El papel del Capital Social en el Desarrollo Económico Local. Revista de Estudios Empresariales. Segunda Época (2): 67-92. Recuperado de http://revistaselectronicas.ujaen.es/index.php/ REE/article/view/366/329.

Merino, L. (2004). Conservación o deterioro. Impacto de las politicas públicas en las instituciones comunitarias y en los usos de los bosques en México. Distrito Federal, México: Secretaría de Medio Ambiente y Recursos Naturales.

Ostrom, E. (1999). Social Capital: A Fad or a Fundamental Concept. En P. Dasgupta e I. Serageldin (eds.). Social Capital A Multifaceted Perspective. Washington, D. C., Estados Unidos: The World Bank.

Ostrom, E., y Toh Kyeong, A. (2003). Una perspectiva del capital social desde las ciencias sociales: Capital social y acción colectiva. Revista Mexicana de Sociología (65): 155-233. Recuperado de http://www.scielo.org.mx/pdf/rms/v65n1/v65nla5.pdf.

Porras, S. (2003). Consideraciones en torno a la confianza y a la colaboración en redes organizacionales. Revista Gestión y Estrategia (24): 206-217.

Prat I Forga, J. M. (2014). La generación del capital social alrededor del turismo industrial en destinos de Cataluña y Escocia. GeoGraphos, 5(58): 47-69.

Putnam, R.; Leonardi, R., y Nanetti, R. (1993). Making Democracy Work. Civic Traditions in Modern Italy. Princeton, Nueva Jersey, Estados Unidos: Princeton University Press.

Putnam, R. (2000). Bowling Alone. The Collapse and Revival of American Community. Nueva York, Estados Unidos: Simon and Schuster Paperbacks.

Real Academia Española (2001). Diccionario de la lengua española. Recuperado de http:// lema.rae.es/drae/srv/search?key=habilidad.

Robison, L. J.; Siles, M. E., y Schmid, A. (2003). El capital social y la reducción de la pobreza: Hacia un paradigma maduro. En R. Atria, M. Siles, I. Arriagada, L. J. Robinson y S. Whiteford (compiladores). Capitalsocialy reducción de lapobreza en América Latina y el Caribe: en busca de un nuevo paradigma (pp. 51-114). Santiago, Chile: Comisión Económica para América Latina y el Caribe, Universidad del 
Estado de Michigan. Recuperado de http://repositorio.cepal.org/bitstream/ handle/11362/2327/S029693_es.pdf?sequence=1\&isAllowed=y.

SAIZ, J. E., y Rangel, S. (2008). Capital social: Una revisión del concepto. Revista CIFE (13): 250-263. Recuperado de https://core.ac.uk/download/pdf/7082270.pdf.

SAndoval, L., y Ortega, M.E. (2016). Implementación de la política pública de Pueblos Mágicos. Caso de estudio: Tapijulapa, Tabasco. Revista Internacional de Ciencias Sociales Interdisciplinares, 5(1). Recuperado de http://journals.epistemopolis.org/ index.php/csociales/article/view/373.

Secretaría de Turismo (s/f). Programa Pueblos Mágicos. Distrito Federal, México:

Secretaría de Turismo. Recuperado de http://www.ordenjuridico.gob.mx/ Publicaciones/CDs2008/CDProgramasyreglas/pdf/45.pdf.

Secretaría de Turismo (s/f). Programa de Desarrollo Regional Turístico Sustentable y Pueblos Mágicos. Diagnóstico del programa presupuestario de SECTUR. Distrito Federal, México: Secretaría de Turismo. Recuperado de http://www.transparenciapresupuestaria.gob.mx/work/models/PTP/Reingenieria_Gasto/imagenes/ Ventanas/Ramo_21/21S248.pdf.

Secretaría de Turismo (2004). Turismo alternativo. Una nueva forma de hacer turismo. Serie Turismo Alternativo, Fascículo 1. Distrito Federal, México: Secretaría de Turismo.

Szauer, M. T., y Castillo, M. S. (2002). Capital social: Articulador de desarrollo sostenible. En F. Jaramillo y M. Szauer (eds.). Capital social. Clave para una agenda integral de desarrollo. Caracas, Venezuela: Unidad de Publicaciones de la Corporación Andina de Fomento.

Van Dijk, T. (1996). Análisis del discurso ideológico. Versión (6): 15-43. Recuperado de http://www.segundaslenguaseinmigracion.com/L2ycomptext/Anlisisideolgico.pdf. 